\title{
A mesoscopic analysis of a localized shear band propagation effect on the deformation and fracture of coated materials
}

\author{
Ruslan Balokhonov ${ }^{1}$, Varvara Romanova ${ }^{1}$ and Aleksandr Zemlianov ${ }^{1}$ \\ ${ }^{1}$ Institute of Strength Physics and Materials Science, Tomsk, Russia
}

\begin{tabular}{l} 
Article Info \\
\hline Article history: \\
Received December 21, 2020 \\
Revised February 2, 2020 \\
Accepted February 5, 2020 \\
\hline
\end{tabular}

\section{Keywords:}

Mesomechanics;

Microstructure-based modeling;

Coated materials;

Lüders band propagation.

\begin{abstract}
The numerical simulations of the deformation and fracture in an iron boride coating - steel substrate composition are presented. The dynamic boundaryvalue problem is solved numerically by the finite-difference method. A complex geometry of the borided coating - steel substrate interface is taken into account explicitly. To simulate the mechanical behavior of the steel substrate, use is made of an isotropic strain hardening model including a relation for shear band propagation. Local regions of bulk tension are shown to arise near the interface even under simple uniaxial compression of the composition and in so doing they determine the mesoscale mechanisms of fracture. The interrelation between plastic deformation in the steel substrate and cracking of the borided coating is studied. Stages of shear band front propagation attributable to the interface complex geometry have been revealed. The coating cracking pattern, location of the fracture onset regions and the total crack length are found to depend on the front velocity in the steel substrate.
\end{abstract}

Copyright (C) 2020 Regional Association for Security and crisis management and European centre for operational research. All rights reserved.

\section{Corresponding Author:}

Ruslan Balokhonov, Institute of Strength Physics and Materials Science, SB, RAS, 634021, Tomsk, Russia.

Email: rusy@ispms.ru

\section{Introduction}

Control over the properties of the surface layer plays an important role in designing modern materials. Coating deposition technologies are widely used in civil engineering, gas and oil industry and agriculture for reconditioning machine parts and devices. Special attention is given to materials for microelectronics, where surface oxidation initiates multiple cracking. The remedy is coating deposition to prolong the service life of components.

Until recently, the most popular structural materials have been surface hardened steels. Depending on the alloy type used as a substrate, different physical processes develop under external loading. Quasi homogeneous plastic flow is commonly observed. However, certain types of steels exhibit an unstable behavior evident as intermittent yielding. For example, experimental stress-strain curves for steels (NematNasser and Guo 2005, Balokhonov and Romanova 2021, Ren et al. 2021) are characterized by upper and lower yield stresses, which bear witness to Lüders band propagation.

The serrated yielding in alloys has been well studied experimentally, for instance, by Beukel and Kocks (1982), Nagornih et al. (1993), Casarotto et al. (2003), Nemat-Nasser and Guo (2005), Belotteau et al. (2009), Ke et al. (2014), Tretyakova et al. (2018), Ren et al. (2021) and is attributed to the formation of localized deformation bands at the mesoscale level. A special case of such an anomalous behavior is Lüders band propagation characterized by a displacement of a macroscopic strain localization zone along the test piece. It is generally agreed that the microscopic cause of the discontinuous yielding is the dynamic aging of 
dislocations by diffusing solute atoms. There are attempts to simulate propagation of localized plastic deformation bands (McCormick and Ling, 1995; Hähner and Rizzi, 2003, Belotteau et al., 2009, Romanova et al. 2011, Ren et al., 2021, Balokhonov et al. 2003, 2021).

Hard and superhard coating depositions, including nanostructured coatings, and investigations of their mechanical, physical and chemical properties have been the object of a great number of papers, see, e.g., a review by Pogrebnyak et al. (2009) or Tkadletz et al. (2016). Particular emphasis has been placed on the microstructure, strength, stiffness, heat and corrosion resistance of coatings. Another important problem is investigations of the properties of a composition as a whole. For instance, it is appropriate to perform microstructure-based numerical simulations of the ability of a material with a coating possessing certain physical-mechanical properties to resist external loading.

Nowadays there are a large number of studies addressing multidimensional numerical simulation and modeling of materials where explicit consideration is given to microstructure. This involves development of adequate constitutive models for components of composite materials. Some of the papers deal with artificial models of real microstructures for studying micro-, mesoscale and homogenized macromechanical response of the material (e.g., Needleman et al. (1985), Llorca et al. (1991), Romanova et al. (2003, 2009, 2011, 2017), Carrere (2004), Balokhonov et al. (1999, 2020), etc.). Other authors report results on a stress-strain analysis of experiment-based microstructure models (see, e.g., Sørensen et al. (1992), Schmauder (2002), Chawla et al. (2006), Balokhonov et al. (2006, 2009, 2019, 2021). This and related research work gives us a better understanding of the relationship between the microstructure and the mechanical properties of materials.

Special attention is given to interfaces. The majority of publications devoted to the interfacial problems considers the interfacial fracture, decohesion and debonding (see, e.g., Needleman (1990), Chandra and Ghonem (2001), Guo et al. (2005)). In our earlier work (Balokhonov et al. 2006, 2009, 2019, 2021), we studied phenomena related to irregular interface geometry effects. Mechanically, stress concentration in local regions of a composite at different scale levels is assumed to be of the same origin. It is controlled by the irregular geometry of microstructure elements making up the composition (ductile matrix/substrate, brittle ceramic inclusions/coatings/hardened interlayers, etc.) and by the difference in their mechanical properties. Local stresses in an aluminum-alumina composite were found to exceed the average level of the load applied by a few factors. This effect is attributed to the presence of inhomogeneities with characteristic sizes corresponding to different scale levels (macro, meso and micro). It was demonstrated that the regions of stress concentration might undergo both compressive and tensile stresses irrespective of the type of external loading. The larger the difference in the mechanical properties of the constituents, the higher the local stress level in the vicinity of inhomogeneities of certain geometry. A distinctive feature of surface-hardened materials is the base material-coating interface. The investigations are performed of special features of deformation and failure of coated material due to formation of local zones of bulk tension in the compression of a composite. The main purpose of this work is to simulate numerically the influence of Lüders band propagation on the mechanical properties of coated materials.

\section{Formulation of the problem}

The mesoscopic numerical analysis of deformation and fracture in a coated material given below employs solutions to a system of equations including laws of conservation of mass and angular momentum of motion, strain relations and constituent equations describing a medium. The dynamic boundary-value problem in the plane strain formulation is solved numerically by the finite-difference method (Richtmyer and Morton 1967, Wilkins 1999). To describe the mechanical behavior of the steel substrate and brittle coating, use is made of a plastic flow model, including isotropic strain hardening and Lüders front propagation, and a fracture model, respectively. The fracture criterion takes into account the difference in the critical strength between different types of local compressive and tensile states. Interface geometries correspond to the configurations found experimentally and are accounted for explicitly in the calculations. 


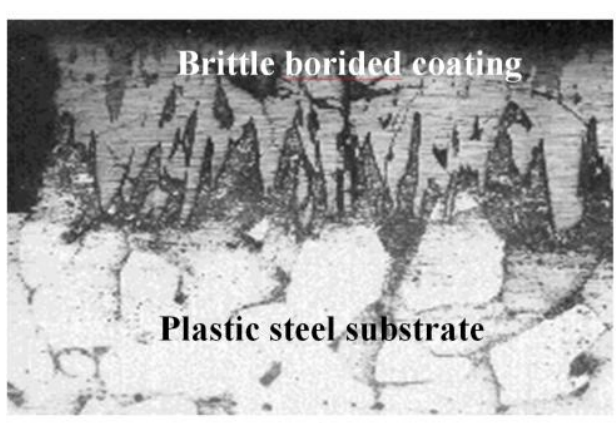

a)

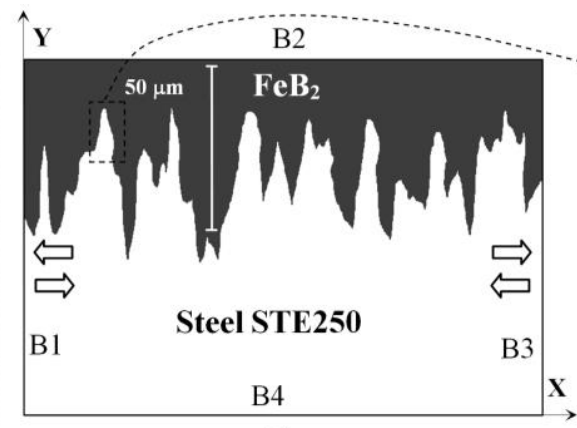

b)

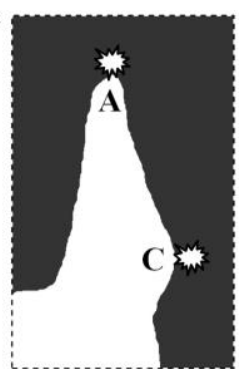

c)

Figure 1. Microstructure of steel STE250: (a) an experiment by Koval and Panin (2000) and (b-c) simulation.

It was found experimentally by Koval and Panin (2000) that a steel - iron boride interface had a characteristic needle-shaped structure (Figure 1a). The evolution pattern of the interrelated processes of deformation of the plastic substrate and cracking of the brittle coating can be demonstrated by simulating the behavior of a simplified two-phase steel-iron boride microstructure. Figure 1c shows the simulated microstructures consisting of a steel substrate and a FeB2 coating corresponding to that observed experimentally.

\subsection{Basic equations and boundary conditions}

Presented in Figure 1 is a lateral face of a flat specimen. Since the specimen size in direction $z$ is about 2 $\mathrm{cm}$, the mechanical behavior of a particular composite microstructure $(250 \times 180 \mu \mathrm{m})$ is simulated using the plane strain formulation.

In the case of plane strain there are the following non-zero components of the strain rate tensor:

$\dot{\varepsilon}_{x x}=\dot{u}_{x, x}, \dot{\varepsilon}_{y y}=\dot{u}_{y, y}, \dot{\varepsilon}_{x y}=\frac{1}{2}\left(\dot{u}_{x, y}+\dot{u}_{y, x}\right)$,

where $u_{x}$ and $u_{y}$ are the components of the displacement vector, $\varepsilon_{x x}, \varepsilon_{y y}$ and $\varepsilon_{x y}$ are the components of the strain tensor, the upper dot and comma in the notations stand for the time and space derivatives, respectively.

The mass conservation law and the equations of motion are of the form

$\dot{V} / V=\dot{\varepsilon}_{x x}+\dot{\varepsilon}_{y y}$,

$\sigma_{x x, x}+\sigma_{y x, y}=\rho \ddot{u}_{x}, \sigma_{x y, x}+\sigma_{y y, y}=\rho \ddot{u}_{y}$,

where $\sigma_{x x}, \sigma_{y y}$ and $\sigma_{x y}$ are the stress tensor components, $V$ is the specific volume and $\rho$ is the mass density.

Allowing for the stress tensor resolution in the spherical and deviatoric parts, we get

$\sigma_{i j}=-P \delta_{i j}+S_{i j}$

the pressure and the stress deviator components are written as follows

$$
\begin{aligned}
& \dot{S}_{x x}=2 \mu\left(\dot{\varepsilon}_{x x}-\frac{1}{3} \dot{\varepsilon}_{k k}-\dot{\varepsilon}_{x x}^{p}\right), \\
& \dot{S}_{y y}=2 \mu\left(\dot{\varepsilon}_{y y}-\frac{1}{3} \dot{\varepsilon}_{k k}-\dot{\varepsilon}_{y y}^{p}\right), \\
& \dot{S}_{z z}=2 \mu\left(-\frac{1}{3} \dot{\varepsilon}_{k k}\right)=-\left(\dot{S}_{x x}+\dot{S}_{y y}\right), \\
& \dot{S}_{x y}=2 \mu\left(\dot{\varepsilon}_{x y}-\dot{\varepsilon}_{x y}^{p}\right), \dot{P}=-K \dot{\varepsilon}_{k k},
\end{aligned}
$$


where $K$ and $\mu$ are the bulk and shear moduli, $\dot{\varepsilon}_{i j}^{p}$ is the plastic strain rate tensor, and $\delta_{i j}$ is the Kronecker delta.

To eliminate an increase in stress due to rigid rotations of local elements, we write deviatoric stresses through the Jaumann derivative

$\dot{S}_{i j}^{*}=\dot{S}_{i j}-S_{i k} \omega_{j k}-S_{j k} \omega_{i k}$,

where $\omega_{i j}=\frac{1}{2}\left(\dot{u}_{i, j}-\dot{u}_{j, i}\right)$ is the material spin tensor.

The strain tensor is the sum of elastic and plastic parts $\varepsilon_{i j}=\varepsilon_{i j}^{e}+\varepsilon_{i j}^{p}$, and $\dot{\varepsilon}_{k k}^{p}=0$ is the hypothesis of plastic incompressibility. Unloading is elastic.

The boundary conditions on the surfaces $B_{1}$ and $B_{3}$ simulate uniaxial tension of a mesovolume parallel to the $\mathrm{X}$-axis, whereas on the bottom and top surfaces, they correspond to the symmetry and the free surface conditions, respectively (Figure 1b). Thus

$\dot{u}_{x}=$ const $=-v$ for $t \geq 0,(x, y) \in B_{1}$,

$\dot{u}_{x}=$ const $=v$ for $t \geq 0,(x, y) \in B_{3}$,

$\sigma_{i j} \cdot n_{j}=0$ for $t \geq 0,(x, y) \in B_{2}$,

$\dot{u}_{y}=0$ for $t \geq 0,(x, y) \in B_{4}$,

$\sigma_{x y}=0$ for $t \geq 0,(x, y) \in B_{1} \cup B_{3} \cup B_{4}$

Here $B=B_{1} \cup B_{2} \cup B_{3} \cup B_{4}$ is the boundary of the computational domain, $t$ is the time, $v$ is the grip velocity and $n_{j}$ is the normal to the surface $B_{2}$.

The system of equations (1-7) are completed with a formula for plasticity $\dot{\varepsilon}_{i j}^{p}$.

\subsection{Plasticity of the steel substrate including an effect of strain aging}

Plastic deformation is described by the flow rule

$\dot{\varepsilon}_{i j}^{p}=\dot{\lambda} \frac{\partial f}{\partial S_{i j}}$

associated with the yield condition

$f\left(S_{i j}\right)=\sigma_{e q}-\sigma_{A}\left(\varepsilon_{e q}^{p}\right)=0$

Here $\lambda$ is a scalar parameter, $\sigma_{e q}$ and $\varepsilon_{e q}^{p}$ are the equivalent stress and equivalent plastic strain

$\sigma_{e q}=\frac{1}{\sqrt{2}} \sqrt{\begin{array}{l}\left(S_{11}-S_{22}\right)^{2}+\left(S_{22}-S_{33}\right)^{2}+ \\ \left(S_{33}-S_{11}\right)^{2}+6\left(S_{12}^{2}+S_{23}^{2}+S_{31}^{2}\right)\end{array}}$,

$\varepsilon_{e q}^{p}=\frac{\sqrt{2}}{3} \sqrt{\begin{array}{l}\left(\varepsilon_{11}^{p}-\varepsilon_{22}^{p}\right)^{2}+\left(\varepsilon_{22}^{p}-\varepsilon_{33}^{p}\right)^{2}+ \\ \left(\varepsilon_{33}^{p}-\varepsilon_{11}^{p}\right)^{2}+6\left(\varepsilon_{12}^{p 2}+\varepsilon_{23}^{p 2}+\varepsilon_{31}^{p 2}\right)\end{array}}$

The function $\sigma_{A}\left(\varepsilon_{e q}^{p}\right)$ prescribes isotropic strain hardening in the steel substrate. The following phenomenological function is used:

$\sigma_{A}=\sigma_{s}-\left(\sigma_{s}-\sigma_{0}\right) \exp \left(-\varepsilon_{e q}^{p} / \varepsilon_{r}^{p}\right)$

where $\sigma_{0}$ is the yield point, $\sigma_{s}$ is the strength and $\varepsilon_{r}^{p}$ is the reference value of plastic strain. 
A phenomenological approach is used to simulate Lüders band propagation. It relies on the experimentally established fact that plastic deformation commences near surfaces and interfaces and then propagates from the surface sources as localized deformation fronts. Therefore, different yield criteria are formulated for surface cells and for local regions in the bulk of the material. In the former case, a local region near the surface becomes plastic if the equivalent stress acting there reaches its critical value, i.e., the only classical force criterion is used for the surface and interface cells. The response of an internal region in the bulk is elastic as long as two conditions are satisfied: the equivalent stress in this cell reaches the yield limit and the plastic deformation accumulated in any of neighboring cells takes on a critical value. Thus, the internal regions are successively involved in plastic deformation by flows propagating from the surface and interface sources.

Mathematically, the classical force criterion given by a constitutive physically-based model developed for any local internal region $D$ is complemented with the necessary condition whereby there is plastic flow at least in one of the regions $D^{*}$ adjacent to $D$ :

$\varepsilon_{e q}^{p^{*}}=\varepsilon_{0}$.

Here $\varepsilon_{0}$ is a new parameter accounting for the material properties associated with the strain aging effects. This is a critical value of the equivalent plastic strain accumulated in the region $D^{*}$ and required for the onset of plastic flow in the region $D$.

\subsection{Fracture of a brittle coating}

A distinctive feature of deformation of brittle materials is the fact that in compression fracture may occur along the planes where stresses are thought to be zero (Kachanov 1974). Thus a crack may propagate along the direction of applied loading, $X$ (Figure 2a). For instance, in was found experimentally that cracks in the reinforcing particles and in the coating of composites subjected to compression are largely oriented along the direction of compression (Balasundaram et al. 2003, Tilbrook et al. 2007). In modeling a homogenous specimen, however, the stress tensor components in transverse direction $Y$ supposed to open the crack are identically zero. Thus, in experiments, cracks propagate under stresses that are zero from the standpoint of mechanics. To describe fracture in this case use is made of deformation criteria. The simplest one is a criterion of positive elongation along $Y$ (Kachanov 1974).

In what follows, in contrast to the case where the material is treated as a homogenous system, we are going to show that in modeling the deformation of composites, the stress tensor components along $Y$ are nonzero. Moreover, there are localized interfacial regions experiencing tensile stresses in external compression of the composite. It is these stresses which give rise to crack opening and growth along the direction of external loading (Figure 2b). A classical analytical solution of elasticity theory for a plate with a round hole can be used for interpreting formation of local tensile regions in compression (Figure 2b).

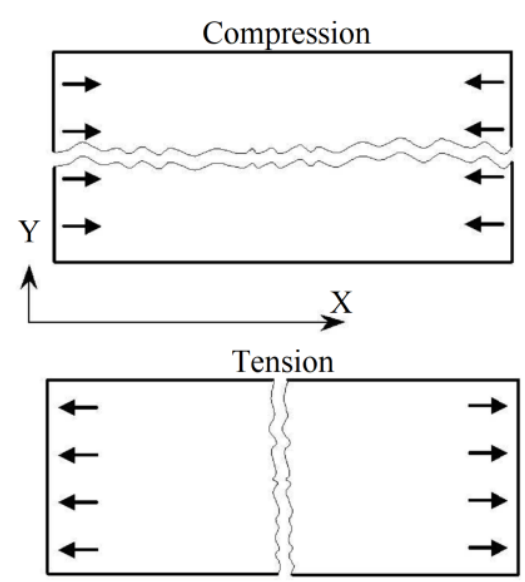

a)

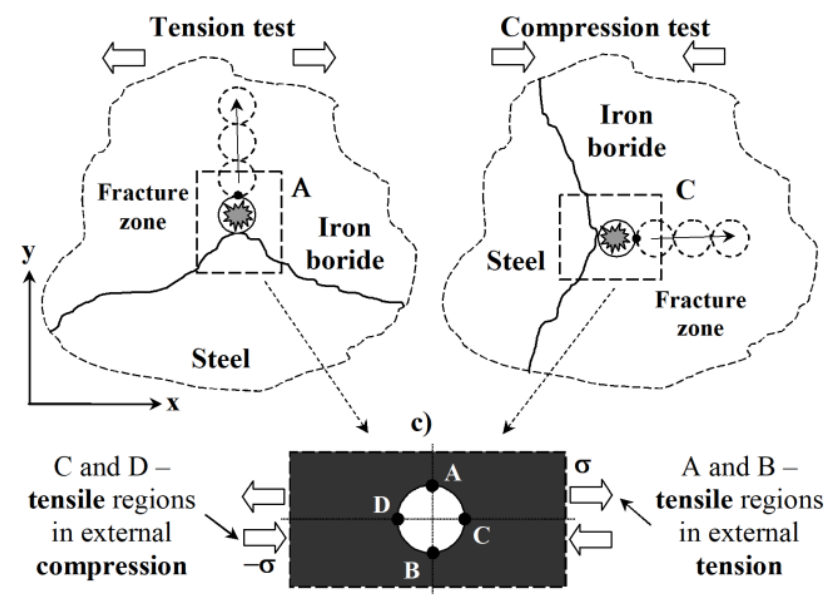

b)

Figure 2. Schematic of crack propagation under different external loading conditions. The macroscopic view (a) and a mesoscopic interpretation of simulation results, using the proposed fracture criterion (b). 
To describe the fracture of the iron boride coating, use was made of a maximum distortion energy criterion. The criterion is thought to poorly describe the fracture of brittle materials. It will be shown below that for real geometry of interfaces, where calculations include localized regions of tensile stresses under any type of external loading, the maximum distortion energy criterion works fairly well and provides a correct description of brittle materials and composites. We have modified the criterion to account for the difference in strength between the tensile and compressive regions:

$\sigma_{e q}=\left\{\begin{array}{l}C_{t e n}, \text { if } \varepsilon_{k k}>0 \\ C_{c o m}, \text { if } \varepsilon_{k k}<0\end{array}\right.$,

where $C_{c o m}$ and $C_{\text {ten }}$ are the tensile and compressive strength.

According to the examined criterion (Eq. (14)), fracture occurs in the local regions undergoing bulk tensile loading. The following fracture conditions are imposed on any local region in the coating: if the cubic strain $\varepsilon_{k k}$ is positive and $\sigma_{e q}$ reaches a critical value $C_{c o m}$, all deviatoric stress tensor components in this region are taken to be zero, and in the case of $\varepsilon_{k k}<0$ and $\sigma_{e q} \geq C_{t e n}$, the pressure $P$ is zero as well.

Table 1. The mechanical properties of the steel substrate and borided coating

\begin{tabular}{cccccccc}
\hline & $\mathrm{K}, \mathrm{GPa}$ & $\mu, \mathrm{GPa}$ & $\sigma_{\mathrm{s}}, \mathrm{MPa}$ & $\sigma_{0}, \mathrm{MPa}$ & $\varepsilon_{\mathrm{r}}{ }^{\mathrm{p}}$ & $\mathrm{C}_{\text {ten }}, \mathrm{GPa}$ & $\begin{array}{c}\mathrm{C}_{\mathrm{com}}, \\
\mathrm{GPa}\end{array}$ \\
\hline $\begin{array}{c}\text { Steel substrate } \\
\text { Borided } \\
\text { coating }\end{array}$ & 60 & data & 497 & 278 & 0.1 & - & - \\
\hline
\end{tabular}

The mechanical properties of the steel substrate and borided coating are given in Table 1 (Koval and Panin 2000; Grigorieva and Meilikhova ed. 1991).

\section{Results and discussion}

\subsection{General behavior of a coated material in tension or compression}

Examine a problem on loading the structure shown in Figure 1 where no account is taken of Lüders band propagation in the steel substrate. Figure 3 illustrates stress-strain curves for specimens subjected to tension or compression. Here and in what follows, the stress $\langle\sigma\rangle$ was computed as the equivalent stress $\sigma_{\text {eq }}$ averaged over a mesovolume $\langle\sigma\rangle=\sum_{k=1, N} \sigma_{e q}^{k} s^{k} / \sum_{k=1, N} s^{k}$, where $N$ is the number of computational mesh cells and $s^{k}$ is the k-th cell area. The strain $\varepsilon$ corresponds to the relative elongation of the computational domain along $\mathrm{X}$ direction $\varepsilon=\frac{L-L_{0}}{L_{0}} \cdot 100 \%$, where $L_{0}$ and $L$ are the initial and current lengths of the mesovolume along $\mathrm{X}$.

The curves exhibit distinct deformation stages in the material characterized by elasticity (region 1 ), plasticity (region 2) and fracture (region 3).

In stage 1 , both the steel substrate and the borided coating undergo elastic strain. The difference in the elastic moduli between the coating and the substrate is responsible for nonuniform stress and elastic strain distribution (Figure 4). The stress concentration along the coating-substrate interface is seen to vary with the shape of the iron boride "teeth" penetrating into the steel substrate and to be at its maximum in regions of the highest interfacial curvature. 


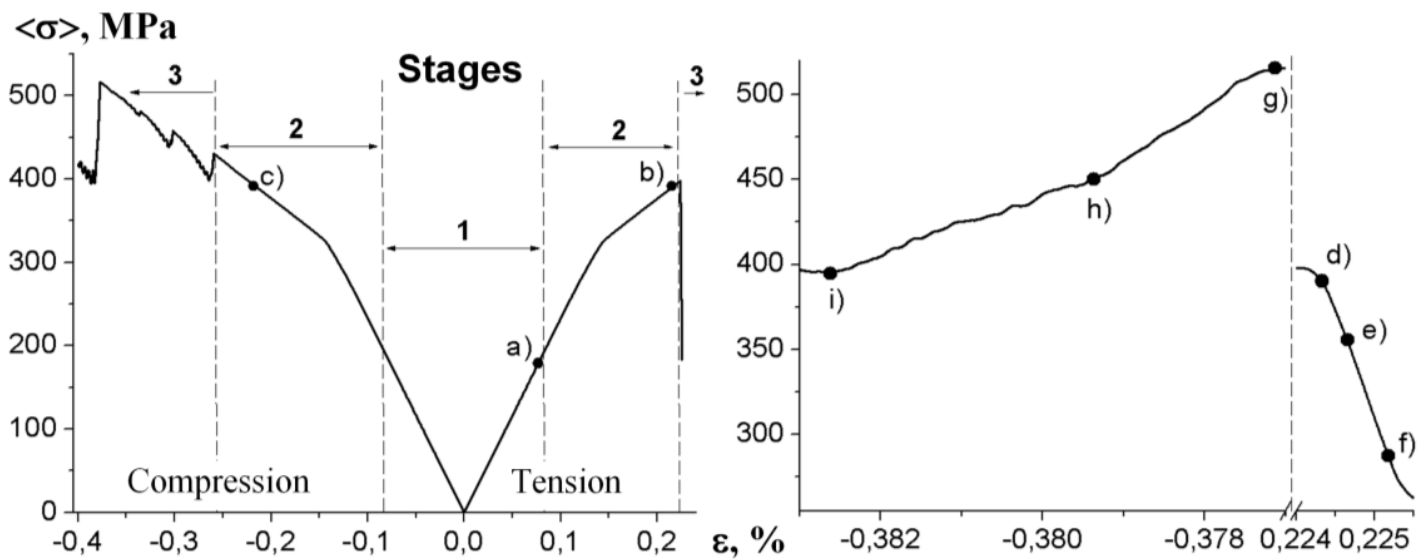

Figure 3. Different stages of the coated material deformation. Stress-strain curves for a coated material and distinct portions corresponding to crack propagation in the coating in tension or compression.

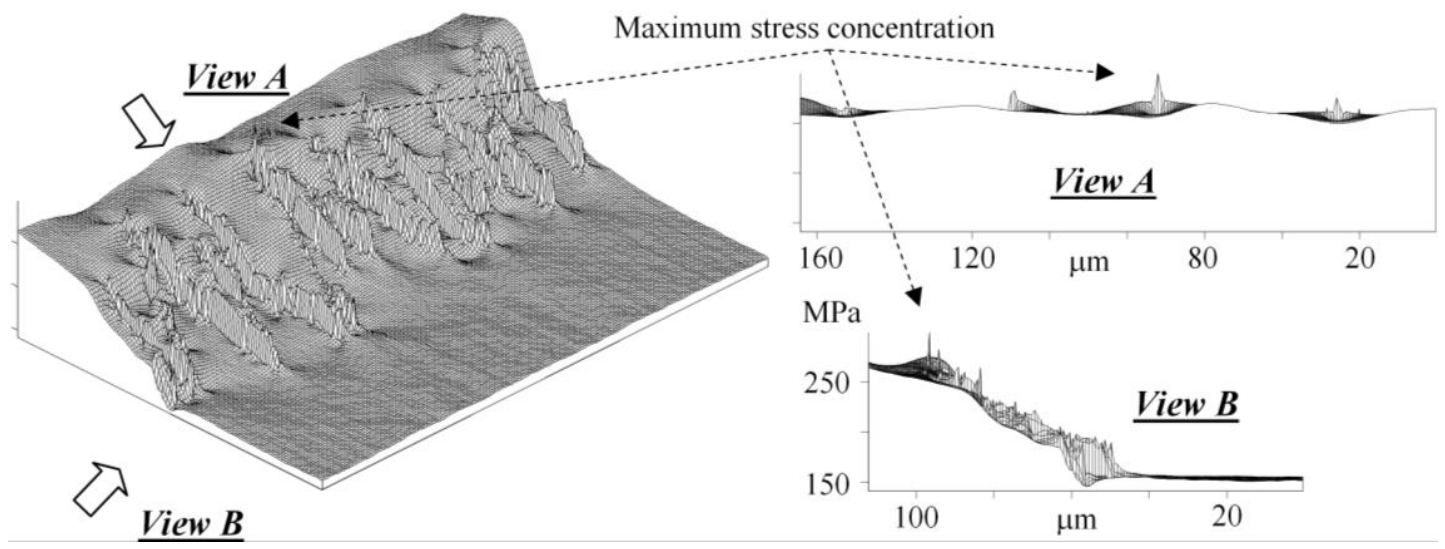

Figure 4. Equivalent stress distribution in the elastic stage (state (a) in Figure 3).

In stage 2, the coating is still in the elastic state, whereas the substrate undergoes plastic strain. Plastic shear strains are generated along the interface in the vicinity of maximum stress concentrations. Further loading gives rise to localized plastic deformation bands. Notably, in view of the fact that the same elasticplastic model is valid for different loading conditions, in stages 1 and 2, the stress and strain tensor components in tension and compression agree qualitatively and quantitatively up to a sign, whereas equivalent stresses and strains are identical. The special features of the deformation behavior of coated materials in stage 2 will be considered in detail in sect. 3.2.

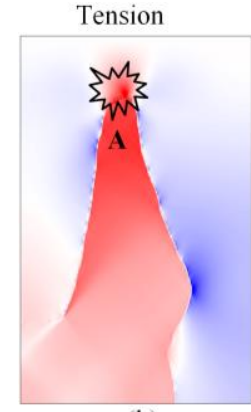

(b)

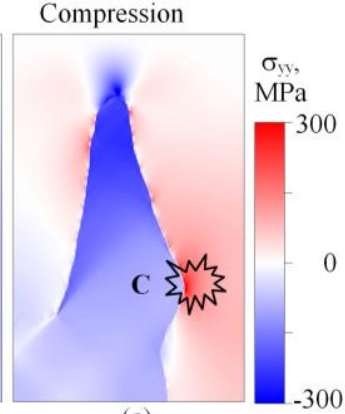

(c)

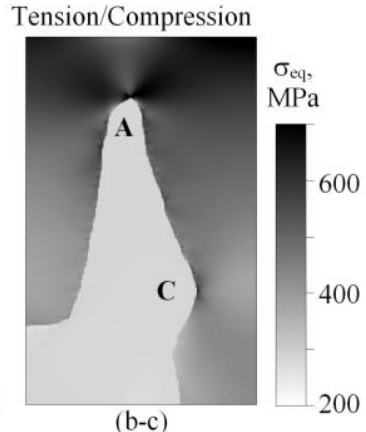

Figure 5. Stress tensor component $\sigma_{y y}$ and equivalent stress $\sigma_{\mathrm{eq}}$ distributions in tension and compression. States $(b)$ and $(c)$ are shown in Fig. 3. 
Stage 3 corresponds to degradation of the composite in question. The interrelated processes of plastic flow in the substrate and cracking of the coating develop simultaneously.

As is seen from Figure 3, the composite subjected to compression exhibits a longer elastic-plastic deformation stage than in tension. In this case, the coating fails at higher degrees of the total strain and at a higher average stress level. The reason is that the crack nucleation in the coating both under applied tensile and compressive loading takes place in local tensile regions, and the crack growth is due to tensile loading. Figure 5 illustrates a stress state for a region of a smaller scale that lies near the root of an iron boride tooth (Figure 1c). The stress tensor components $\sigma_{y y}$ are shown for the tensile prefracture stage (state $(b)$ in Figure 3). Also shown in Figure 5 are the pertinent $\sigma_{y y}$ distribution for compression (state $(c)$ in Figure 3 ) and the equivalent stress distribution. The latter pattern is the same for states $(b)$ and $(c)$ in tension and compression.

The results obtained lead us to conclude that (1) the tensile regions lie in different zones in tension and compression - zones of the $A$ and $C$ types, respectively (Figure 1c), and (2) a maximum equivalent stress concentration in both cases is observed in zones of the $A$ type (Figure $5 b-c$ ).

Thus, under applied compressive loading, zones of the $C$ type (see Figures (1) and (5)) located along the interface are exposed to lower tensile loads than zones of the $A$ type (Figures (1) and (5)) in tension. This is why local fracture zones are formed in the coating at a higher average stress level in compression than in tension, and cracks propagate at a lower velocity in the former case. These findings are clearly demonstrated in Figure 3, where descending portions of the stress-strain curves associated with crack growth are shown on an enlarged scale. In compression, the curve is seen to lie higher and its slope is more gentle than in tension. This is evidence of a lower crack growth rate in compression than in tension.
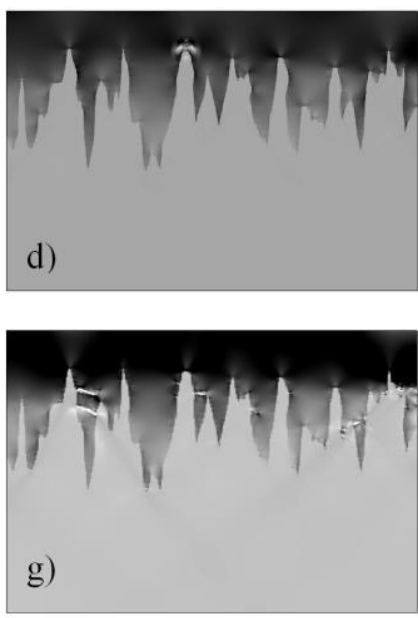
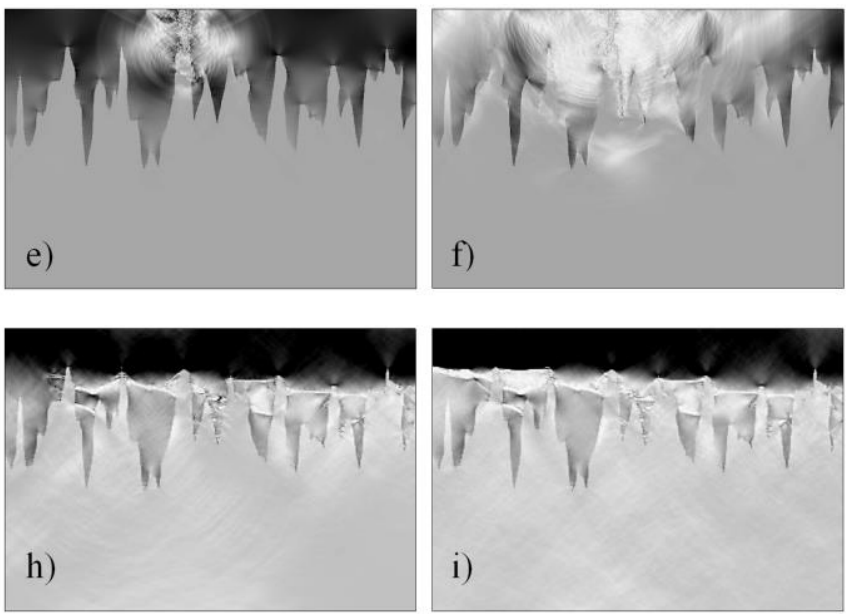

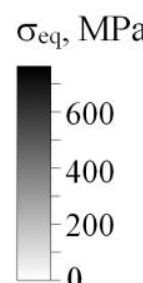

Figure 6. Equivalent stress distribution $\sigma_{\mathrm{eq}}$ in tension and in compression. States $(d-i)$ are shown in Figure 3.

In tension, a single crack appears near the coating-substrate interface (Figure 6d) and propagates toward the specimen surface transversely to the direction of load application (Figure 6e). Crack nucleation is a dynamic process resulting in the formation of a free surface from which release waves propagate to unload the coating material (Figure 6f). The descending portion in the stress-strain curve in Figure 3 is evidence of the fact that specimen loses its strength at the macroscale level.

Compression gives rise to multiple cracking. The first cracks originate in zones of the $\mathrm{C}$ type and propagate toward opposite lateral faces of the iron boride teeth (Figure $6 \mathrm{~g}$ ). Since the cracks spread along the direction of load application, adjacent stress concentration regions are not unloaded. On further loading other cracks are formed there (Figure 6h). The interface is of a characteristic needle shape, which is why the main longitudinal crack is not formed. Only the serrated interlayer fails, whereas the coating surface layer continues to resist loading (Figure 6i). While descending portions are seen in the stress-strain curve, the average stress level does increase (Figure 3). 


\subsection{The special features of plastic flow with consideration for Lüders band propagation}

Let us consider in more detail the stage 2 deformation of the composite. Different types of steel can be used as substrates, where a Lüders band may or may not propagate. The two cases are denoted in the figures below as $L B$ and $N L B$, respectively.

Both in the $L B$ and in the $N L B$ cases, the plastic deformation stage in the substrate can be divided into 4 substages. The plastic flow pattern in each of the substages differs fundamentally in the cases under consideration. Figures 7-9 show simulation results of relevance to our discussion.

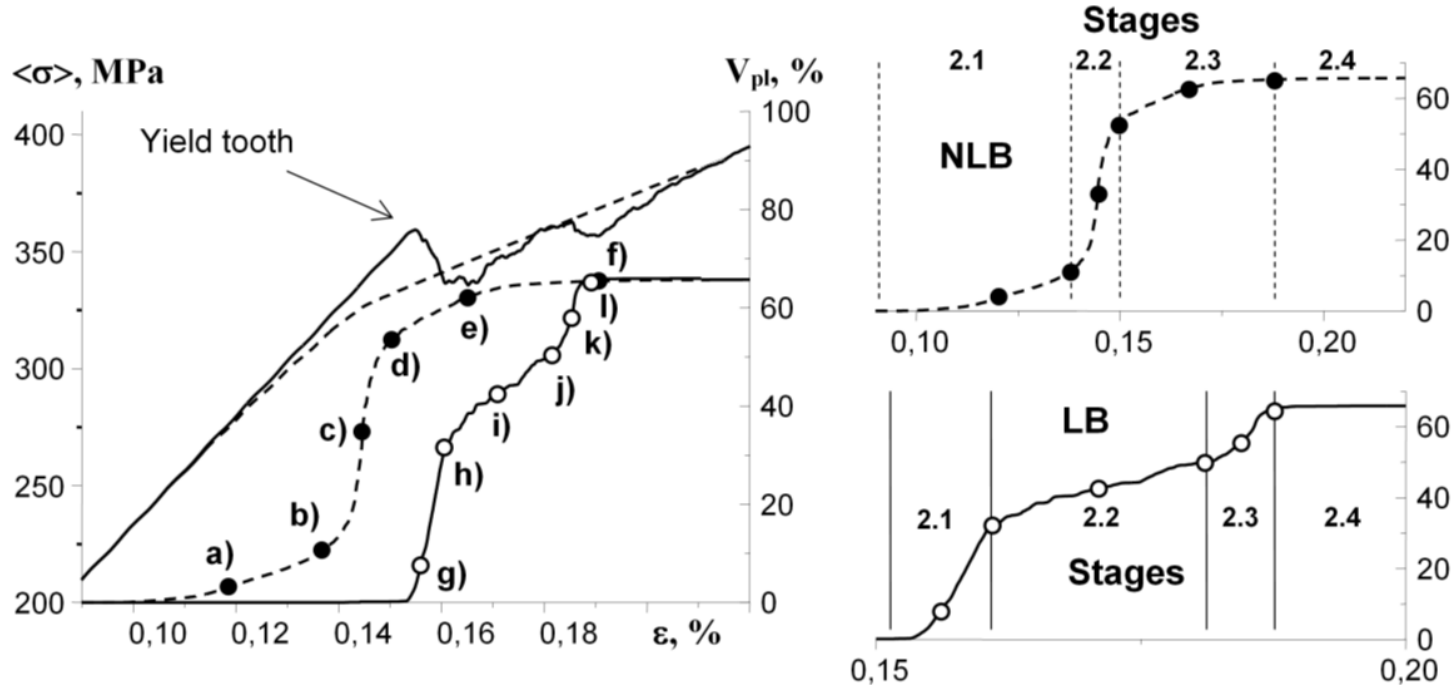

Figure 7. Different stages of plastic flow in the steel substrate. Portions of the stress-strain curves and the volume of the plastically strained steel substrate material: solid curves correspond to the $L B$ case, whereas dotted curves depict the $N L B$ case. $\varepsilon_{0}=2 \cdot 10^{-4}$.

In the case of no Lüders band propagation, the stress increases monotonically. The slope of the stressstrain curve changes, as the steel substrate transforms into a plastic state (Figure 7). Plastic shear strains are initially observed in the vicinity of stress concentrators near the root of the iron boride teeth (Figure 8a). As loading is increased, plastic strain penetrates deep into the specimen, propagating gradually in the regions of the steel base lying between the iron boride teeth (Figure 8b). Thus, the plastic flow in stage 2.1 (Figure 7) is localized in the vicinity of the iron boride teeth that serve as stress concentrators, whereas most of the substrate undergoes elastic deformation. In stage 2.2, the stress concentrators in the vicinity of the iron boride teeth points generate localized shear bands (Figure $8 \mathrm{c}-\mathrm{d}$ ). The shear bands propagate in the direction of maximum tangential stresses at an angle of $\approx 45^{\circ}$ to the loading axis. In stage 2.3 , plastic flow involves the regions of the material lying between the bands (Figure 8e-f). On further loading the average level of the equivalent plastic strain increases (stage 2.4 in Figure 7). It is evident from the figure that the plot of the volume of the material involved in plastic flow, $\mathrm{V}_{\mathrm{pl}}$ against the degree of strain exhibits a maximum slope between points $(b)$ and $(d)$. Stage 2.2 is the shortest one. In this stage, most of the substrate transforms into a plastic state, the velocity of propagation of plastic strains is at a maximum and the slope of the macroscopic stress-strain curve changes abruptly.

A fundamentally different nonhomogeneous strain pattern is observed in the case where a Lüders band is assumed to propagate in the substrate material $(L B)$. The stress-strain curve exhibits well-pronounced serrations and a characteristic yield drop (Figure 7). Plastic strains initially appear in the vicinity of a stress concentrator where the left computational domain boundary crosses the coating-substrate interface (Figure $9 \mathrm{~g}$ ). A Lüders band front propagating in the direction of load application toward the opposite boundary of the mesovolume is formed. The front moves at a variable velocity, and different stages are seen as well (Figure 7). In contrast to the $N L B$ case, the Lüders front propagates at a maximum velocity in stage 2.1 and covers about $50 \%$ of the substrate material (Figures 7 and $9 \mathrm{~g}-\mathrm{h}$ ). A yield tooth is seen in the stress-strain curve. Then the propagation of the Lüders band slows down (Figures 7 and $9 \mathrm{~h}-\mathrm{j}$ ). In stage 2.2, the composite is stretched both due to a slow movement of the front and to an accumulation of plastic strain behind the front. In stage 2.3, the Lüders band is accelerated to reach the right computational domain boundary, and the plastic flow covers the entire steel substrate (Figures 7 and $9 j-l$ ). The serrations in the homogenized stress are attributed to a nonuniform motion of the Lüders band determined by a complex configuration of the coating- 
substrate interface. To the rapid propagation of the Lüders front (stages 2.1 and 2.3) correspond the descending portions of the stress-strain curve in Figure 7.

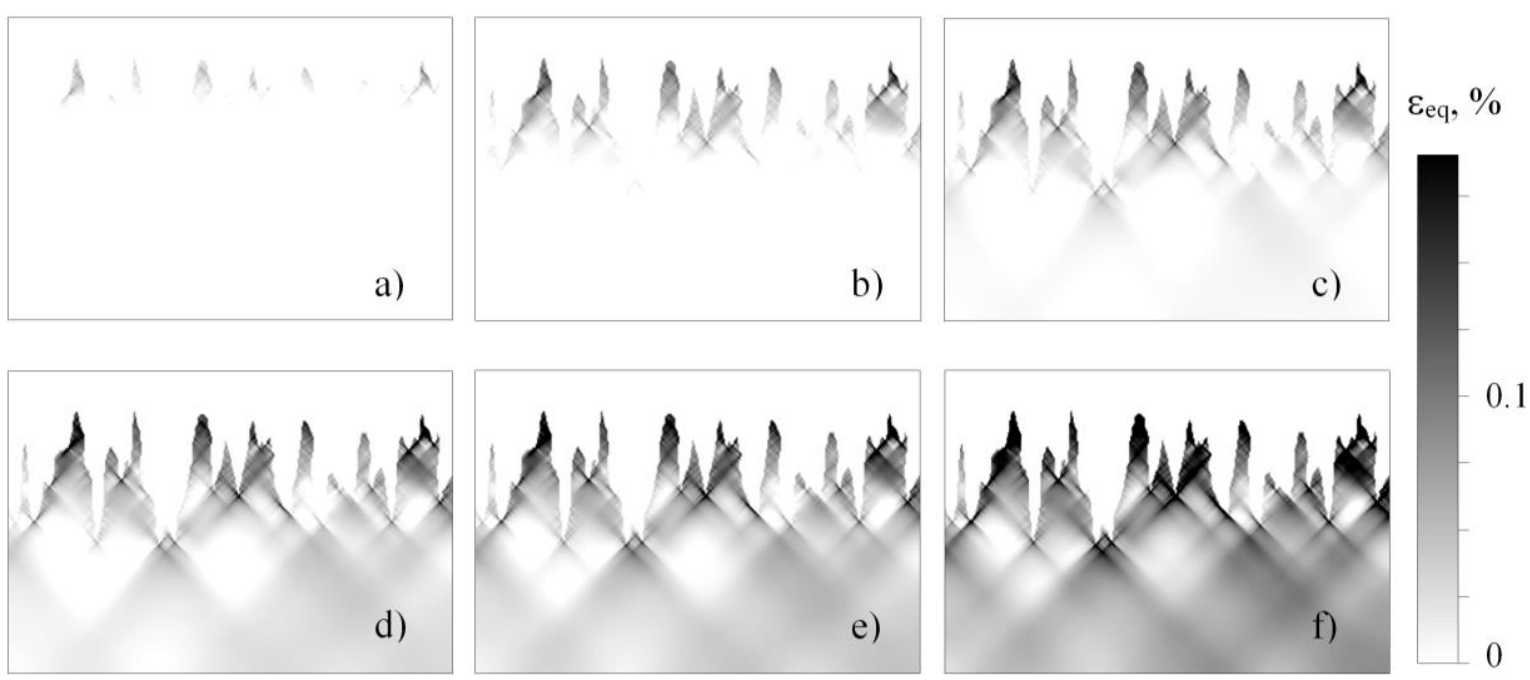

Figure 8. Equivalent plastic strain distribution with Lüders band propagation being ignored $(N L B)$. States $(a-f)$ are shown in Figure 7.
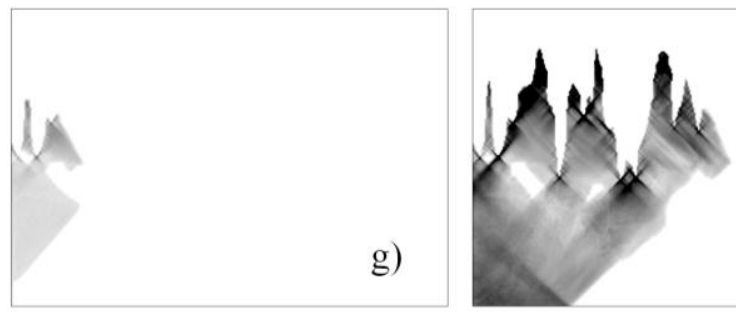

h)
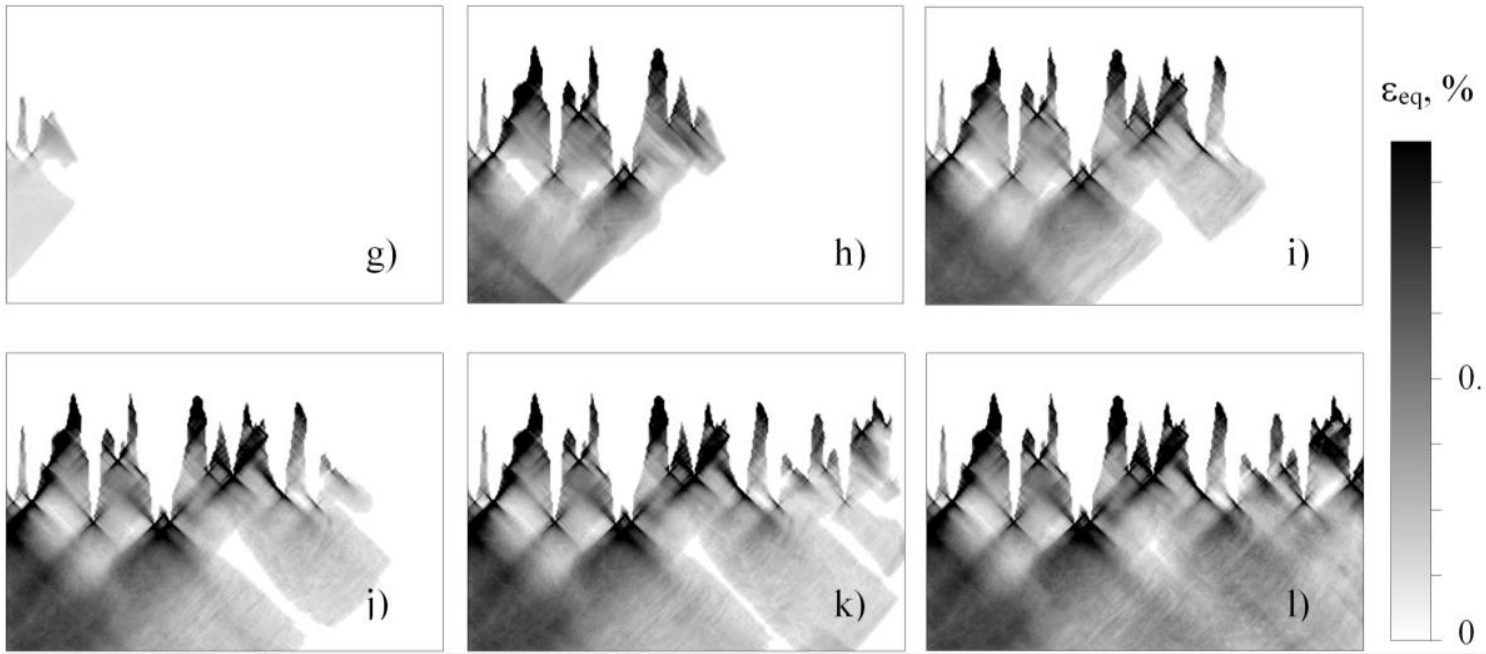

0.1

Figure 9. Equivalent plastic strain distribution allowing for Lüders band propagation $(L B)$. States $(g-l)$ are shown in Figure 7.

On passage of the Lüders band (the $L B$ case) equivalent plastic strain distribution agrees, on the whole, both qualitatively and quantitatively with the distribution in the $N L B$ case (Figures $8 f$ and $9 l$ ). In stage 2.4, the substrate is deformed in a quasi-uniform manner: only the average level of the equivalent plastic strain increases, whereas the distribution pattern changes but slightly. At some time in the loading process, stress jumps disappear and the $N L B$ and $L B$ stress-strain curves meet each other. A classical stage of the strain hardening curve is realized in both cases (Figure 7). 


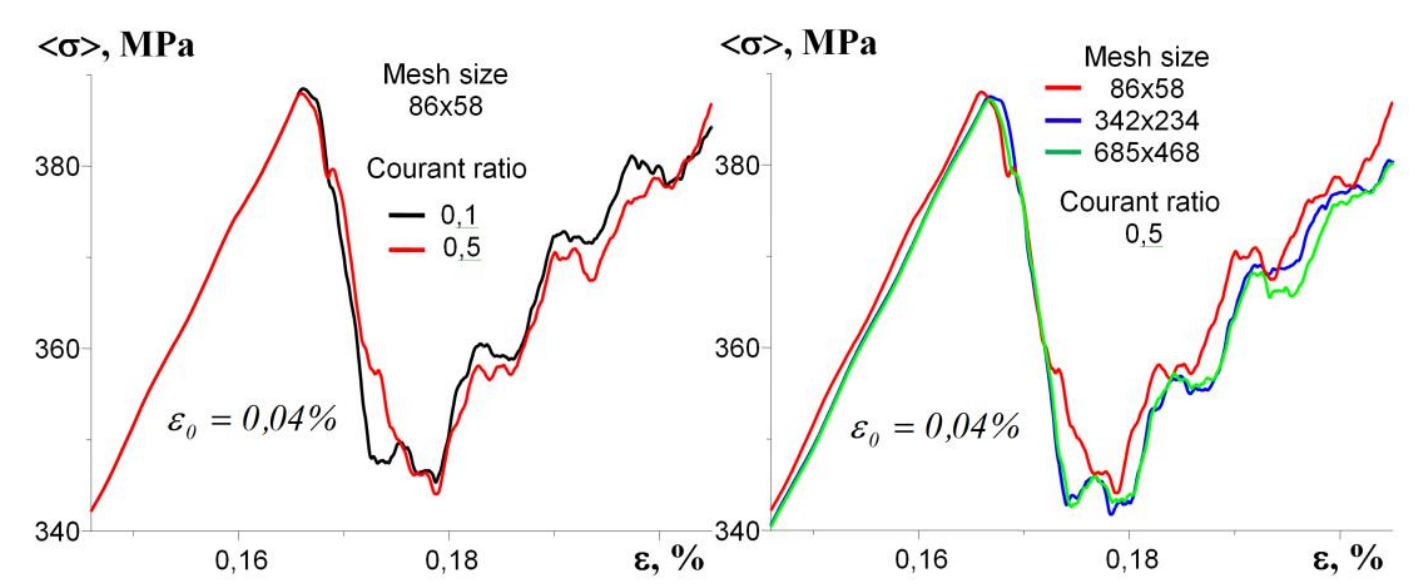

Figure 10. Portions of stress-strain curves for varying Courant ratio $(a)$ and mesh size $(b)$.

$$
\varepsilon_{0}=4 \cdot 10^{-4} \text {. }
$$

We have investigated the convergence of the numerical solution for the Lüders band propagation. The calculations of the mesovolume tension were performed for varying Courant ratio and step in space (Figure 10). Model (13) is seen to provide good convergence of the calculation results.

\subsection{Relationship between fracture and plastic flow}

On further loading of the composite the coating fails. Let us consider the interplay between fracture of the borided coating and plastic deformation of the substrate as a Lüders band propagates in the base material.

It is of interest to investigate the effect of the parameter $\varepsilon_{0}$ (see Eq. (13)). The physical meaning of $\varepsilon_{0}$ is related to the dynamic aging of dislocations by solute atoms. The higher is $\varepsilon_{0}$, the higher is the energy it takes a dislocation to overcome the barrier and continue to move.

The stress-strain curves for the composite subjected to tension or compression are plotted in Figure 11 for varying $\varepsilon_{0}$.

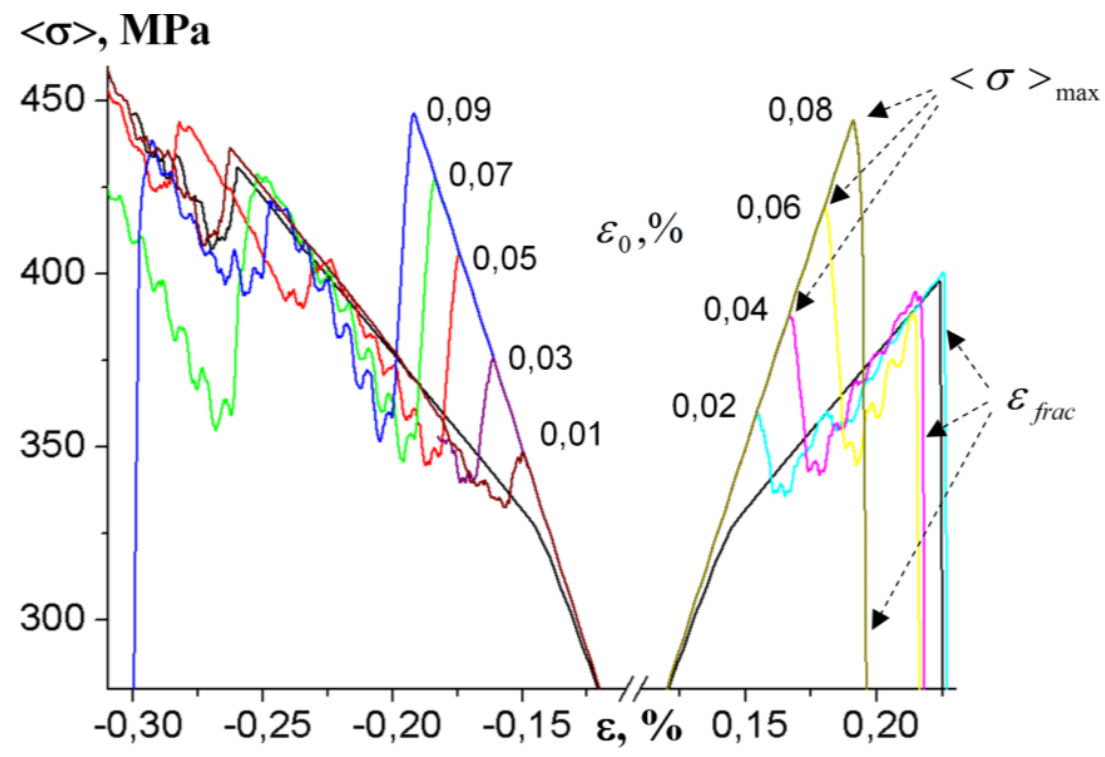

Figure 11. Stress-strain curves for different values of $\varepsilon_{0}$.

Let us examine 3 possible scenarios depending on the values of $\varepsilon_{0}:$ (1) a Lüders band propagates through the entire substrate and then the coating fails, (2) motion of the Lüders band is accompanied by fracture of 
the coating, and (3) the fracture of the coating takes place in the stage where the steel substrate undergoes elastic deformation.

The simulation results suggest the following conclusions. In tension, the maximum value of the yield stress $\langle\sigma\rangle_{\max }$ (Figure 11) is linearly dependent on $\varepsilon_{0}$ for scenarios 1 and 2 and does not change for scenario 3 (Figure 12a). The composite strain at the fracture onset $\varepsilon_{\text {frac }}$ (Figure 11) changes nonmonotonically with $\varepsilon_{0}$ for scenario 2 and remains unchanged for scenarios 1 and 3 (Figure 12b).

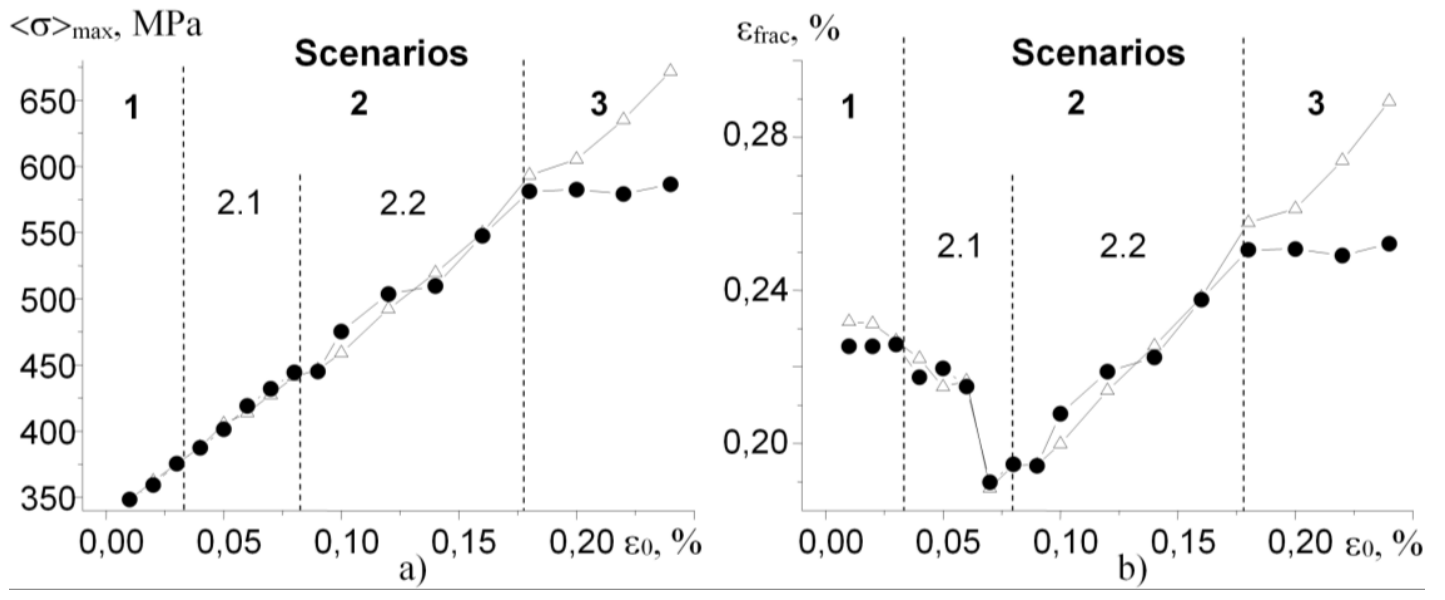

Figure 12. Different scenarios of the relationship between fracture and plastic flow according to different ranges of values of $\varepsilon_{0}$. Maximum stress a) and strain of the composition at the onset of

fracture b) vs. $\varepsilon_{0}$. The circles and triangles illustrate tension and compression, respectively.

For scenario $1,\langle\sigma\rangle_{\max }$ has a meaning of the upper yield point. For low values of $\varepsilon_{0}$, the plastic flow in the steel substrate is followed by cracking of the coating, and $\langle\sigma\rangle_{\max }$ increases linearly with $\varepsilon_{0}$ (Figure 12a). Initially, a Lüders band propagates from the left to the right boundary of the computational domain. This gives rise to a nonuniform pattern of the plastic strain tensor components. However, the stress concentration distribution near the interface is, on the whole, in qualitative and quantitave agreement with the NLB case (Figure 9f, 91). Therefore, the subsequent crack nucleation in the coating takes place in the same region as in the NLB case, and the cracking pattern remains unchanged (Figure $6 \mathrm{f}, 13 \mathrm{a}$ ). The value of $\varepsilon_{\text {frac }}$ is fixed (Figure 12b) and is determined by the stress concentration near the elastic coating - plastic substrate interface.
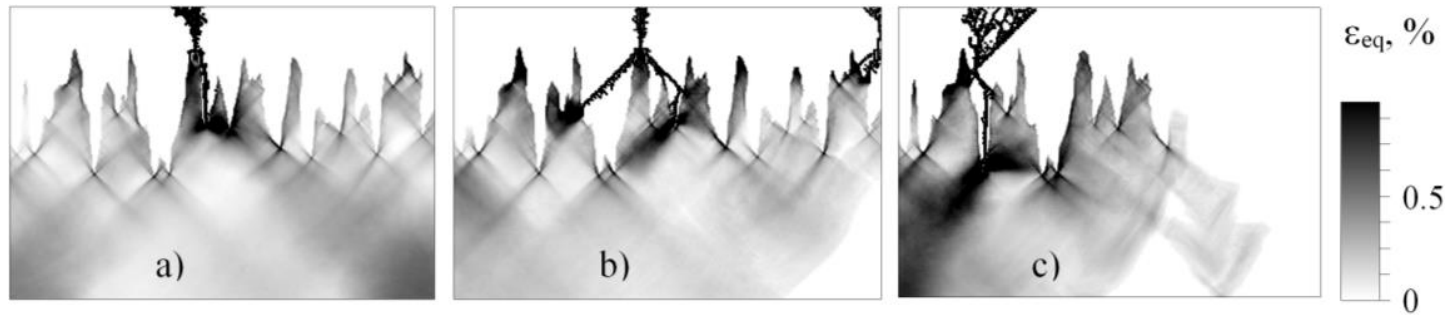

Figure 13. Equivalent plastic strain patterns for a total tensile strain $\varepsilon=0.22 \%$ (see Figure 11) for varying $\varepsilon_{0}, \%$ : a) $\left.\left.-0.02, \mathrm{~b}\right)-0.04, \mathrm{c}\right)-0.06$. Fractured regions in the coating are shown in

black.

For moderate values of $\varepsilon_{0}$, the coating fails during Lüders band propagation. This scenario 2 is of interest for investigating the interplay between plastic flow in the substrate and fracture in the coating. The value of $\varepsilon_{0}$ influences the cracking pattern and determines the region of the coating material where fracture will take place (Figure 13), because the moving Lüders band causes redistribution of the stress concentrators 
near the interface. Scenario 2 is characterized by two ranges of values of $\varepsilon_{0}$. For the first case (scenario 2.1 in Figure 12), the coating fails (Figure 13b, c) in the Lüders band propagation stages 2.2-2.4 (Figure 7). That is why $\langle\sigma\rangle_{\max }$ has a meaning of the upper yield point as in the case of scenario 1 and $\varepsilon_{\text {frac }}$ decreases with $\varepsilon_{0}$ (Figure 12b). For scenario 2.2 (Figure 12), the coating cracks in stage 2.1 of rapid propagation of the Lüders band. In this case the stress drop in the stress-strain curve is due to propagation of the plastic flow front in the substrate and to crack growth in the coating. Now the maximum homogenized stress $\langle\sigma\rangle_{\max }$ has a combined meaning of the upper yield point and ultimate strength. Due to the latter contribution to $\langle\sigma\rangle_{\max }$ that remains, on the whole, a linear monotonic function of $\varepsilon_{0}$ (Figure 12a), $\varepsilon_{\text {frac }}$ starts to increase with $\varepsilon_{0}$.

At very high values of $\varepsilon_{0}$, there is no Lüders band propagation in the substrate. The stress drop in the stress-strain curve is completely due to cracking of the coating material. $\langle\sigma\rangle_{\max }$ has a meaning of the strength of the composition, is determined by the stress concentration near the elastic coating - elastic substrate interface and is independent of the plastic strain $\varepsilon_{0}$. Accordingly, $\varepsilon_{\text {frac }}$ ceases to change for scenario 3 (Figure 12b). Is should be noted that scenario 3 can be realized in the case where high-strength doped alloys are used as substrates. These materials, as a rule, are characterized by high elastic moduli and yield point and by finite plasticity. What may happen in such a situation is that crack nucleation in the brittle coating will take place in the elastic stage of the substrate deformation. In the case at hand, however, high values of $\varepsilon_{0}$ lead to overestimated strength. This effect has not been detected in experiments on soft steel of the STE250 type. In addition, high values of $\varepsilon_{0}$ mean that dislocation detachment from impurities occurs at high local stresses. This assumption calls for special physical substantiation as well.

Similar conclusions can be arrived at for compressive loading of the composition, except that scenario 3 is shifted to higher values of $\varepsilon_{0}$. This is due to the fact that compression crack nucleation takes place in local regions exposed to lower loads than in the tension regime (see sect. 3.1).
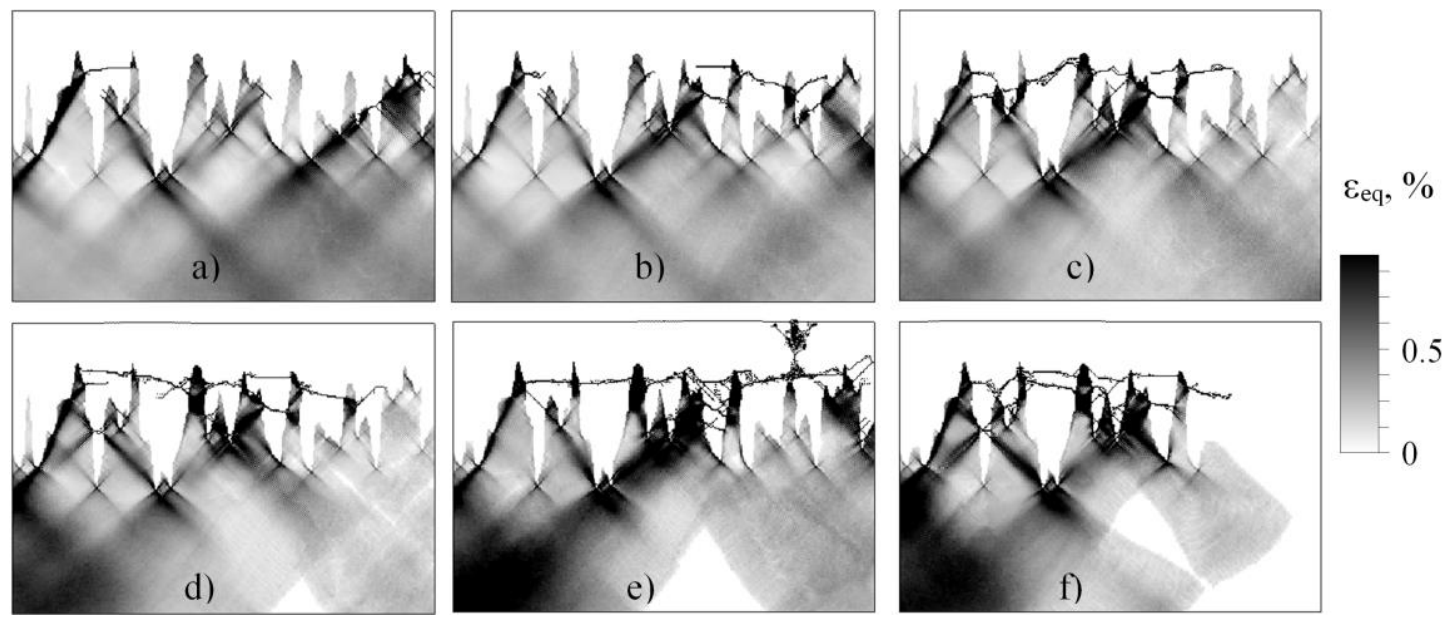

0.5

Figure 14. Equivalent plastic strain patterns for a total compressive strain $\varepsilon=-0.3 \%$ (see Figure 11) for different values of $\varepsilon_{0}, \%$ : a) $\left.\left.\left.-0.05, \mathrm{~b}\right)-0.06, \mathrm{c}\right)-0.07, \mathrm{~d}\right)-0.08$, e) $\left.-0.09, \mathrm{f}\right)-$

0.1 . Fractured regions in the coating are shown in black.

Unlike tensile loading, where a single crack propagates from the interface to the specimen surface and provides unloading of the composition in the direction of load application, compression gives rise to multiple cracking of the coating. In other words, several cracks are generated in succession and propagate in the direction of compressive loading.

The calculations for a composition subjected to compression have revealed that the higher is $\varepsilon_{0}$, the lager is the number of cracks in the coating (Figure 14). The volume content of the failed material was found to be an exponentially increasing function of $\varepsilon_{0}$ (Figure 15, black circles). This conclusion holds true for scenario 1. It is also valid for scenario 2 on condition that the Lüders band covers the entire substrate. For moderate 
values of $\varepsilon_{0}$ (scenario 2), however, an inverse dependence is observed in the case where the Lüders band continues to move: $V_{\mathrm{ep}}$ decreases with increasing $\varepsilon_{0}$ (Figure $15, \varepsilon_{0}>0.08$, triangles). This is due to the fact that cracks appear for the most part behind the moving front. Plastic flow develops there, and the stress concentration near the elastic coating - plastically strained steel substrate is higher than that found near the elastic coating - elastically strained steel substrate interface ahead of the Lüders front. The higher is $\varepsilon_{0}$, the lower is the velocity of propagation of the Lüders band and, consequently, the smaller is the region of the steel substrate involved in the plastic flow, and the smaller is the number of cracks in the coating.

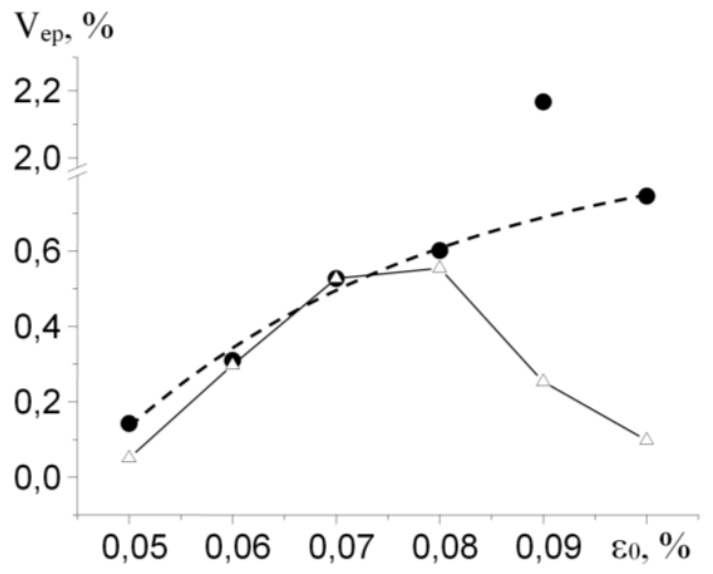

Figure 15. Volume content of the failed borided coating vs $\varepsilon_{0}$. The circles and triangles illustrate different values of the compressive strain $\varepsilon=-0.3 \%$ and $\varepsilon=-0.27 \%$, respectively.

It should be kept in mind that in compression of the composition all cracks are generated in local regions of bulk tension and propagate under the action of tensile loads (see sect. 3.1). The Lüders front moving at a variable velocity for different values of $\varepsilon_{0}$ is responsible for a redistribution of stress concentrators and for changes in the stress-strain state. That is why, for certain values of $\varepsilon_{0}$, equivalent stress in a local region of the material exposed to external compressive loading may exceed the critical compressive value $C_{c o m}$. In this case, a crack is formed that propagates transversely to the axis of loading (Figure 14e) and causes a loss in strength of the composition at the macroscale level (Figure 11, $\varepsilon_{0}=0.09$, blue curve), just as in the case of a tension crack. Due to the change in the cracking mechanism, the volume of the failed material dramatically increases and becomes anomalously high (Figure 15, $\varepsilon_{0}=0.09$, black circle).

\section{Conclusions}

The mechanisms of deformation and fracture in a steel substrate-iron boride coating composition subjected to uniaxial tension and compression were investigated. A dynamic boundary-value problem was solved numerically by the finite-difference method. Based on a maximum distortion energy criterion a fracture model that takes into consideration the difference in the critical strength between local tensile and compressive regions was proposed. It provides an adequate description of the direction of crack propagation in brittle materials and composites where explicit account is taken of real geometry of interfaces. To describe Lüders front propagation attributed to the dynamic aging of dislocations by diffusing solute atoms at the microscale level, the yield criterion was modified by introducing a new parameter that has a meaning of the critical plastic strain $\varepsilon_{0}$. Physically, the parameter is related with the energy it takes a dislocation to overcome the solute atom barrier. Different stages of nonhomogeneous plastic flow were revealed in the cases with and without Lüders band propagation in the coated steel. The stages are associated with complex geometry of the coating-substrate interface. A series of numerical experiments was carried out for varying $\varepsilon_{0}$.

The results of the numerical simulations suggest the following conclusions. 
1. Cracks originate and propagate predominantly in the regions of local tension under any type of external loading. In the course of external tension or compression, cracks nucleate in different zones and grow in different directions.

2. The Lüders front velocity varies during deformation of the composition. Early in the plastic flow the front exhibits the highest velocity. This gives rise to the formation of a yield tooth and yield plateau on the stress-strain curve. The averaged Lüders band velocity decreases with increase in $\varepsilon_{0}$.

3. The upper yield point linearly increases with $\varepsilon_{0}$, whereas macroscopic fracture strain is a nonmonotonic function of $\varepsilon_{0}$. This result is due to the interrelated processes of Lüders front propagation in the substrate and cracking of the coating.

4. The higher is the value of $\varepsilon_{0}$, the more intensive is the cracking of the coating. The volume of the failed material depends exponentially on $\varepsilon_{0}$.

Acknowledgement: The work was performed according to the Government research assignment for ISPMS SB RAS, project FWRW-2021-0002.

\section{References}

Balasundaram, A., Gokhale, A. M., Graham, S., Horstemeyer, M. F. (2003). Three-dimensional particle cracking damage development in an Al-Mg-base wrought alloy. Materials Science and Engineering: A, 355, 368-383.

Balokhonov, R. R., Makarov, P. V., Romanova, V. A. and Smolin, I. Yu. (1999). Simulation of crystal plasticity under dynamic loading. Computational Materials Science, 16 (4), 355-361.

Balokhonov, R. R., Romanova, V. A., Schmauder, S. and Makarov, P.V. (2003). Simulation of meso-macro dynamic behavior using steel as an example. Computational Materials Science, 28, 505-511.

Balokhonov, R., Romanova, V., Schmauder, S. (2006). Computational analysis of deformation and fracture in a composite material on the mesoscale level. Computational Material Science, 37, 110-118.

Balokhonov, R. R., Romanova, V. A. (2009). The effect of the irregular interface geometry in deformation and fracture of a steel substrate-boride coating composite. International Journal of Plasticity, 25, 11, 22252248.

Balokhonov, R., Romanova, V. (2019) On the problem of strain localization and fracture site prediction in materials with irregular geometry of interfaces. Facta Universitatis. Series: Mechanical Engineering, 17 (2), 169-180.

Balokhonov, R., Romanova, V., Kulkov, A. (2020). Microstructure-based analysis of deformation and fracture in metal-matrix composite materials. Engineering Failure Analysis, 110, 104412.

Balokhonov, Ruslan R. and Romanova, Varvara A. (2021). Microstructure-Based Computational Analysis of Deformation and Fracture in Composite and Coated Materials Across Multiple Spatial Scales. In G.P.Ostermeyer et al. (eds.), Multiscale Biomechanics and Tribology of Inorganic and Organic Systems (pp. 377-419). Springer Tracts in Mechanical Engineering.

Belotteau, J., Berdin, C., Forest, S., Parrot, A., Prioul, C. (2009). Mechanical behavior and crack tip plasticity of a strain aging sensitive steel. Materials Science and Engineering: A, 526, 1-2, 156-165.

Beukel, A. V. D., Kocks, U. F. (1982). The strain dependence of static and dynamic strain-aging. Acta Metallurgica et Materialia, 30, 1027-1034.

Casarotto, L., Tutsch, R., Ritter, R., Weidenmüller, J., Ziegenbein, A., Klose, F., Neuhäuser, H. (2003). Propagation of deformation bands investigated by laser scanning extensometry. Computational Materials Science, 26, 210-218.

Carrere, N., Valle, R., Bretheau, T., and Chaboche, J.-L. (2004). Multiscale analysis of the transverse properties of Ti-based matrix composites reinforced by SiC fibres: from the grain scale to the macroscopic scale. International Journal of Plasticity, 20, 783-810. 
Chandra, N., Ghonem, H. (2001). Interfacial mechanics of push-out tests: theory and experiments. Composites Part A: Applied Science and Manufacturing, 32, 575-584.

Chawla, N., Sidhu, R. S., Ganesh, V. V. (2006). Three-dimensional visualization and microstructure-based modeling of deformation in particle-reinforced composites. Acta Materialia, 54, 1541-1548.

Grigorieva, I. S., Meilihova, E. Z., (Eds.). (1991). Physical values. Reference book. Energoatomizdat, Moscow.

Guo, L.-C., Wu, L.-Z., Zeng, T., Cao, D.-h. (2005). The transient response of a coating-substrate structure with a crack in the functionally graded interfacial layer. Composite Structures, 70, 109-119.

Hähner, P., Rizzi, E. (2003). On the kinematics of Portevin-Le Chatelier bands: theoretical and numerical modeling. Acta Materialia 51, 3385-3397.

Kachanov, L. M. (1974). Fundamentals of fracture mechanics. Nauka, Moscow.

Ke, H. B., Sun, B. A., Liu, C. T., Yang, Y. (2014). Effect of size and base-element on the jerky flow dynamics in metallic glass, Acta Materialia, 63, 180-190.

Koval, A. V., Panin, S. V. (2000). Mesoscale deformation and cracking of surface-hardened low carbon steel. Theoretical and Applied Fracture Mechanics, 34, 117-121.

Llorca, J., Needleman, A., Suresh, S. (1991). An analysis of the effects of matrix void growth on deformation and ductility in metal-ceramic composites. Acta Metallurgica et Materialia, 39, 2317-2335.

McCormick, P. G., Ling, C. P. (1995). Numerical modeling of the Portevin-Le Chatelier effect. Acta Metallurgica et Materialia, 43, 1969-1977.

Nagornih, S. N., Sarafanov, G. F., Kulikova, G. A. (1993). Plastic deformation instability in cooper alloys. Russian Physics Journal, 36, 112-117.

Needleman, A., Asaro, R. J., Lemonds, J., Peirce, D. (1985). Finite element analysis of crystalline solids. Computer Methods in Applied Mechanics and Engineering, 52, 689-708.

Needleman, A. (1990). An analysis of tensile decohesion along an interface. Journal of the Mechanics and Physics of Solids, 38, 289-324.

Nemat-Nasser, S., Guo, W. G. (2005). Thermomechanical response of HSLA-65 steel plates: experiment and modeling. Mechanics of Materials, 37, 379-405.

Panin, S. V., Durakov, V. G., Pribitkov, G. A. (1998). Mesomechanics of plastic deformation and fracture of low-carbon steel with high-strength coating. Physical Mesomechanics, 1, 51-58.

Pogrebnyak, A. D., Shpak, A. P., Azarenkov, N. A., and Beresnev, V. M. (2009). Structure and properties of hard and superhard nanocomposite coatings, Physics-Uspekhi, 52 (1), 29-54.

Ren, S. C., Morgeneyer, T. F., Mazière, M., Forest, S., Rousselier, G. (2021). Effect of Lüders and PortevinLe Chatelier localization bands on plasticity and fracture of notched steel specimens studied by DIC and FE simulations. International Journal of Plasticity, 136, 102880.

Richtmyer, R. D., Morton, K. W. (1967). Difference methods for initial-value problems. Interscience publishers, John Wiley \& Sons, New York - London - Sydney.

Romanova, V., Balokhonov, R., Makarov, P., Schmauder, S., and Soppa, E. (2003). Simulation of elastoplastic behaviour of an artificial 3D-structure under dynamic loading. Computational Materials Science, 28, 518-528.

Romanova, V., Balokhonov, R., Schmauder, S. (2009). The influence of the reinforcing particle shape and interface strength on the fracture behavior of a metal. Acta Materialia, 57, 97-107.

Romanova, V., Balokhonov, R., Schmauder, S. (2011). Three-dimensional analysis of mesoscale deformation phenomena in welded low-carbon steel. Materials Science and Engineering: A, 528, 5271-5277.

Romanova, V., Balokhonov, R., Panin, A., Kazachenok, M., Kozelskaya, A. (2017). Micro- and mesomechanical aspects of deformation-induced surface roughening in polycrystalline titanium. Materials Science and Engineering: A, 697, 248-258. 
Schmauder, S. (2002). Computational Mechanics. Annual Review of Materials Research, 32, 437-465.

Sørensen, N., Needleman, A., Tvergaard, V. (1992). Three-dimensional analysis of creep in a metal matrix composite. Materials Science and Engineering: A, 158, 129-137.

Tretyakova Tatyana, Zubova Ekaterina, (2018). Influence of additional vibration impact on kinetics of strain bands due to the Chernov-Lüders deformation and Portevin-Le Chatelier effect in metals. Procedia Structural Integrity, 13, 1739-1744.

Tilbrook, M. T., Paton, D. J., Xie, Z., Hoffman, M. (2007). Microstructural effects on indentation failure mechanisms in TiN coatings: Finite element simulations. Acta Materialia, 55, 2489-2501.

Tkadletz Michael, Schalk Nina, Daniel Rostislav, Keckes Jozef, Czettl Christoph, Mitterer Christian. (2016). Advanced characterization methods for wear resistant hard coatings: A review on recent progress. Surface and Coatings Technology, 285, 31-46.

Wilkins, Mark L. (1999). Computer simulation of dynamic phenomena. Springer. 